\title{
In vivo MR spectroscopy and MR imaging on non-anaesthetized marine fish: techniques and first results
}

\author{
Christian Bock*, Franz-Josef Sartoris, Hans-Otto Pörtner \\ Alfred-Wegener-Institute for Polar and Marine Research, Columbusstraße, D-27568 Bremerhaven, Germany
}

Received 21 November 2001; accepted 27 January 2002

\begin{abstract}
In vivo Magnetic Resonance Imaging (MRI) and Spectroscopy (MRS) experiments were carried out in non-anaesthetized marine fish with a maximum body length of $40 \mathrm{~cm}$ using a $4.7 \mathrm{~T}$ horizontal magnet. In flow-through animal chambers long-term investigations could be carried out for several days in a sessile benthic zoarcid species, the eelpout Zoarces viviparus, as well as in a pelagic gadid species, the cod Gadus morhua. Sea water adapted probes and optimised excitation and refocusing pulse shapes reduced the negative effects of the highly conductive medium. In both species, in vivo ${ }^{31} \mathrm{P}-\mathrm{NMR}$ spectra were collected within 5 minutes with a reasonable signal to noise ratio ( $\mathrm{PCr} / \mathrm{Pi}$ ratio $>40$ in cod) and typical line widths in the range of 20 to max. $40 \mathrm{~Hz}$. Magnetic resonance images were recorded in the sessile species without movement artefacts. In these fish it was even possible to measure the blood flow of different vessels with a flow compensated gradient echo sequence and to carry out localized in vivo ${ }^{1} \mathrm{H}-\mathrm{NMR}$ spectroscopy in different organs. (C) 2002 Elsevier Science Inc. All rights reserved.
\end{abstract}

Keywords: In vivo MRI; MRS; Marine fish; Comparative physiology

\section{Introduction}

In vivo Magnetic Resonance methods become more and more applicable to study physiological problems in animal models in environmental research. Especially for comparative studies in aquatic animals the use of in vivo NMR spectroscopy has increased (for a review see [1]). However, in vivo $\mathrm{MR}$ imaging studies are still very rare (e.g. [2,3]). As MRI scanners become more accessible for biological researchers and comparative physiologists, MR imaging and spectroscopy experiments are feasible to study metabolism or organ functions in organisms other than typical medical animal models like rats and mice. This allows to address physiological phenomena in organisms subjected to a wide range of environmental influences and thereby, to come to a deeper understanding of biochemical and physiological adaptations in animals, which are often extremists in solving

\footnotetext{
* Corresponding author. Tel.: +49-471-4831-1288; fax: +49-4714831-1149.

E-mail address: cbock@awi-bremerhaven.de (C. Bock).
}

physiological problems compared to mammalian animal models. This poses methodological problems of NMR methods have to be well adapted to the environmental factors of these "new" models, e.g. study of animals that live in fresh or sea water, sediment or at extreme or constant temperatures like the permanent cold of the polar seas.

Especially in marine organisms, there are only a few NMR studies available. Already in 1990 Blackband and Stoskopf [4] presented the first successful in vivo MR images and spectra from marine organisms. Since then, the number of potential applications to marine organisms has increased, allowing to study temperature adaptation [5,6], osmoregulation [7], the effect of toxicants including natural products that even can help to answer medical questions. The present paper outlines an MR imaging and spectroscopy approach in unrestrained and non-anaesthetized marine fish, which allows long-term investigations under welldefined physiological conditions. Results are presented that have been obtained in benthic marine fish, where almost no problems with movement artefacts occurred. This approach has been extended to free-swimming pelagic fish. A perspective section deals with possible applications in future studies on marine animals. 


\subsection{Practical considerations and methods}

The main prerequisite for in vivo NMR experiments is the maintenance of well-defined and controlled physiological conditions inside the magnet. In the case of marine organisms measurement periods over several days or weeks may be required, since metabolic changes in some species may only develop over days and will stabilize very slowly [8].

\subsection{Design of the animal chambers, experimental set-up}

The limited space inside an NMR system determined by the magnet bore size and the fact that sea water with its high ion content and conductivity negatively affects the performance of the radio-frequency coils (RF-coils), especially at higher magnetic field strength, restricts the amount of sea water to be used in NMR experiments. Blackband and Stoskov [4] suggested a flowing multi-compartment water system. We adapted this set-up and modified it for the special purposes of higher magnetic field strength (in our case $4.7 \mathrm{~T}$ ) and smaller magnet bores (40 cm diameter) (see $[5,6]$ ). Perspex animal chambers (Rietzel, Hannover, Germany) of variable length and diameter were developed to support the animals inside the magnet. Water exchange was provided by recirculation (made of Tygon tubings) from a temperature controlled sea water reservoir (80 1 of natural sea water), which contained a recirculated aquarium filter for water purification. The reservoir was equilibrated with air by use of a gas-mixing pump, which even allowed the defined change of gas partial pressures (Wösthoff, Bochum, Germany). Water flow through the animal chamber was maintained by hydrostatic pressure and could easily be regulated with a valve placed directly behind the reservoir. Water flow driven by a hydrostatic pressure difference has the advantage that the flow is not influenced by pump induced pressure pulses. Furthermore, RF-noise from unscreened pumps can be avoided. After passing the chamber the sea water was collected in a bin and transported back to the reservoir by a peristaltic pump. This set-up allowed direct in vivo NMR measurements at different but constant flow speeds without the necessity to switch off the flow during MR acquisition. Temperature control was maintained by cooling the water reservoir and the support tubings. Temperature in the animal chamber and inside the reservoir was monitored by use of a fiber-optic thermometer (Luxtron 504, Polytec, Waldheim, Germany) and stored to computer disk via a MacLab system (AD-Instruments, Australia). Temperature stability over time was $\pm 0.5^{\circ} \mathrm{C}$ over a temperature range of $0^{\circ}$ to $30^{\circ} \mathrm{C}$.

\subsection{NMR coils}

The salinity and paramagnetic ions of natural sea water decrease the quality factor (Q) of the NMR coils, whereas the main effect results from the conductivity of sea water $[4,9]$. We compared the signal-to-noise ratios ( $\mathrm{S} / \mathrm{N}$ ratio) from samples of natural sea water with artificial solutions without any paramagnetic ions (e.g. manganese) on our experimental set-up and found no differences between them, confirming the finding of Blackband \& Stoskov [4], that conductivity and not paramagnetic ions reduces the $\mathrm{Q}$ of the RF-coils (data not shown). Since these small amounts of paramagnetic ions have no effect on the performance of our NMR coils, but may have an effect on delicate marine animals, we decided to work with natural sea water. As mentioned above the conductivity of sea water decreases $Q$ and therefore induces a loss in $\mathrm{S} / \mathrm{N}$ ratio. In particular, the tuning and matching properties of the coil changes with conductivity ([9], about 50\% reflection and a shift of $3 \mathrm{MHz}$ using our cod chamber filled with sea water in comparison to fresh water) and reduces the $\mathrm{S} / \mathrm{N}$ ratio. By optimising the capacity of the match and tune capacitors in the NMR coil circuit, it is possible to minimize the negative effects of highly conductive media. In our experiments we used sea water adapted NMR coils (Bruker Medical, Ettlingen, Germany) that can be adjusted to a wide range of salt concentrations up to 2 Osmol (conductivity around $90 \mathrm{mS} / \mathrm{cm}$ ). Usually, a $20 \mathrm{~cm}$ diameter ${ }^{1} \mathrm{H}$ birdcage resonator and a $5 \mathrm{~cm}$ diameter triple tuneable ${ }^{1} \mathrm{H}_{-}{ }^{31} \mathrm{P}-{ }^{13} \mathrm{C}$ surface coil were oriented perpendicularly to each other and were operated in a cross coil mode to increase the $\mathrm{S} / \mathrm{N}$ ratio. Furthermore, the surface coil, which could be positioned on the animal chamber near to various body parts of the animals, was used for spectroscopy studies (similar to [6]). Pulse length and consequently the RF-power of the RF-pulses changed dramatically with salinity (up to $11 \mathrm{~dB}$ in our set-ups). After optimisation and correction of the $90 \%$ $180^{\circ} \mathrm{RF}$-pulses spin echo MR images from the animal chamber filled with sea water showed a reduction of less than $10 \%$ in $\mathrm{S} / \mathrm{N}$ ratio in comparison to MR images from the chamber filled with fresh water (data not shown).

\subsection{Animals}

Benthic sessile eelpout Zoarces viviparus from North Sea (ca 15-20 cm, ca $50 \mathrm{~g}$ ) and Baltic Sea (ca $20 \mathrm{~cm}$, ca $60 \mathrm{~g}$ ) were caught by local fishermen in the summer months of 1998 and kept in sea water aquaria at $12^{\circ} \mathrm{C}$ (salinity $34 \%$, respectively $18 \%$ o for Baltic species). Pelagic cod Gadus morhua (30-40 cm, 200-400 g) were caught in the German bight near Helgoland by bottom trawl in the summer of 1998 and kept since then in sea water tanks. Animals were fed once a week with frozen mussels, except for the last week before NMR experimentation. After the experiments the animals were transported back to the aquarium. All animals survived the normal experimental procedure.

\section{NMR methods}

\subsection{MR imaging}

Pilot scans were collected right before every experiment using a standard multi slice gradient echo sequence in all 
three directions to control the position of the animal (matrix: $128 \times 128$ units, FOV $=12 \mathrm{~cm}^{2}$, slice thickness: $5 \mathrm{~mm}$, $\mathrm{TR}=100 \mathrm{~ms}, \mathrm{TE}=10 \mathrm{~ms}$, scan time $25 \mathrm{~s})$.

Multi slice RARE imaging [10] (matrix $128 \times 128$, FOV $=18 \times 11.5 \mathrm{~cm}^{2}, 6$ slices of $2 \mathrm{~mm}$ thickness, slice separation $5 \mathrm{~mm}, \mathrm{TR}=1024 \mathrm{~ms}, \mathrm{TE}=11.4 \mathrm{~ms}$, rare factor 16,8 averages, acquisition time $2.35 \mathrm{~min}$ ) and gradient echo imaging (FLASH [11]; matrix $256 \times 192$, FOV $=18 \times$ $11.5 \mathrm{~cm}^{2}$, 4 slices, $2 \mathrm{~mm}$ slice thickness, flip angle $22.5^{\circ}$, $\mathrm{TR}=100 \mathrm{~ms}, \mathrm{TE}=5 \mathrm{~ms}, 2$ averages, acquisition time 1.16 min) were performed for a comparison of the methods on cod.

For anatomical studies on sessile eelpout multi-slice RARE imaging was performed in coronal directions (matrix: $256 \times 192, \mathrm{FOV}=12 \mathrm{~cm}^{2}, 4$ slices with a slice thickness of $1 \mathrm{~mm}, \mathrm{TR}=2000 \mathrm{~ms}, \mathrm{TE}=10 \mathrm{~ms}$, rare factor of 8 , scan time $1.5 \mathrm{~min}$ ).

For dynamic MR studies flow weighted imaging was performed on eelpout using a flow compensated gradient echo sequence (FLASH). Typical parameters were as follows: matrix $128 \times 128$, FOV $=4 \mathrm{~cm}^{2}, 1$ transversal slice, slice thickness $2 \mathrm{~mm}$, flip angle $80^{\circ}, \mathrm{TR}=100 \mathrm{~ms}, \mathrm{TE}=$ $10 \mathrm{~ms}$, scan time $1.4 \mathrm{~min}$ for 4 averages.

\subsection{NMR spectroscopy}

Localized ${ }^{1} \mathrm{H}-\mathrm{NMR}$ spectra were obtained using a PRESS sequence (voxel size: $5 \mathrm{~mm}^{3}, 6 \times 8 \times 10 \mathrm{~mm}^{3}$ in liver tissue, $\mathrm{TR}=1000 \mathrm{~ms}$, $\mathrm{TE}=27-40 \mathrm{~ms}$, excitation pulse: sinc 3 of $3000 \mu$ s length, refocusing pulse: mao4_180 of $6000 \mu$ s length (sinc3 for liver), ns: 128-512 scans (1024 for liver), size 1024). Water suppression was applied by the CHESS method consisting of three gauss pulses (pulse length $15000 \mu \mathrm{s}$ ).

In vivo ${ }^{31} \mathrm{P}-\mathrm{NMR}$ spectra were acquired continuously over 600-1200 scans, consisting of a $100 \mu \mathrm{s}$ bp pulse, a repetition delay of $0.52 \mathrm{~s}$ resulting in a measurement time of 5 or 10 minutes, respectively. All spectra were processed automatically applying a user program with size $=16 \mathrm{k}$, line broadening $=10 \mathrm{~Hz}$ and an automatic baseline correction. The spectra were calibrated to phosphocreatine (PCr) as an internal standard. The line width of the PCr signal was in the range of $20-40 \mathrm{~Hz}$.

\section{Results and discussion}

The uniform flow, driven by hydrostatic pressure, supported high-resolution MR recordings in sessile as well as pelagic fish. Experiments were carried out over one week under control conditions with animals in a stable physiological condition, demonstrating the excellent water quality and long term stability of environmental parameters (e.g. aeration, temperature).

Interestingly, the animals showed no escape responses during the experiments in spite of acoustic vibrations from the gradient coils over the time course of image acquisition.

The basis of standard MR imaging sequences consists of a spin echo or a gradient echo refocusing part. One difference between both methods is, that gradient echo sequences are very sensitive to local magnetic field changes or inhomogeneities, because spins, which were de-phased by local field inhomogeneities, will not be refocused by gradient echos in contrast to spin echos. This sensitivity of the gradient echo method is now usually used for brain activation studies $\left(\mathrm{T}_{2}{ }^{*}\right.$-weighted or BOLD MR imaging) or can be used for the visualisation or quantification of flow (e.g. MR angiography). The comparison of coronal MR images recorded with both methods is shown in Fig. 1. MR images were collected from a restrained cod under control conditions at a flow rate of $1.2 \mathrm{l} / \mathrm{min}$. This set up was used for it took the maximum volume inside the probe and had the major impact on MR sequences and hardware. The figures on the left were collected with a spin echo sequence (RARE, [10]), whereas the figures on the right were sampled with a standard gradient echo sequence (FLASH, [11]). The artefacts in the FLASH images resulted from sea water flow during the switching of the phase gradient. The RARE images showed no movement artefacts, a signal from surrounding sea water did not appear at all in this case, because of the high flow rate $(1.2 \mathrm{l} / \mathrm{min})$ in comparison to the repetition time. Since no signal of the surrounding water signal could be detected, the dynamic intensity range of the images solely depends upon the signal of the fish tissue. This leads to a higher $\mathrm{S} / \mathrm{N}$ ratio and a high image contrast compared to the FLASH images. Furthermore, no influences of eddy currents or the accompanied skin effect could be observed in contrast to MR images collected without sea water flow. Therefore, anatomical coronal MR images were usually collected with spin-echo sequences. Nevertheless, gradient echo images could be acquired without flow artefacts for transversal slices, if the phase gradient was oriented perpendicular to the flow direction (see below). In this way even dynamic gradient echo imaging techniques like flow weighted and $\mathrm{T}_{2}{ }^{*}$-weighted imaging could be applied.

\subsection{Sessile benthic animals}

Fig. 2 presents coronal anatomical MR images obtained with RARE from a pregnant species of the North-sea eelpout Zoarces viviparus. The anatomical resolution is good enough to distinguish between muscle tissue, gills, spine, stomach and the uterus with embryonic fish. The flow through the chamber was around 0.7 1/min, again, as in Fig. 1 , almost no effect of water could be detected in the image except for some regions behind the body of the animal, where some water flow was blocked (see arrows). A bar was inserted to support the orientation of the animal inside the chamber relative to the surface coil. Although the fish was not anaesthetised and free to move, the animal maintained 

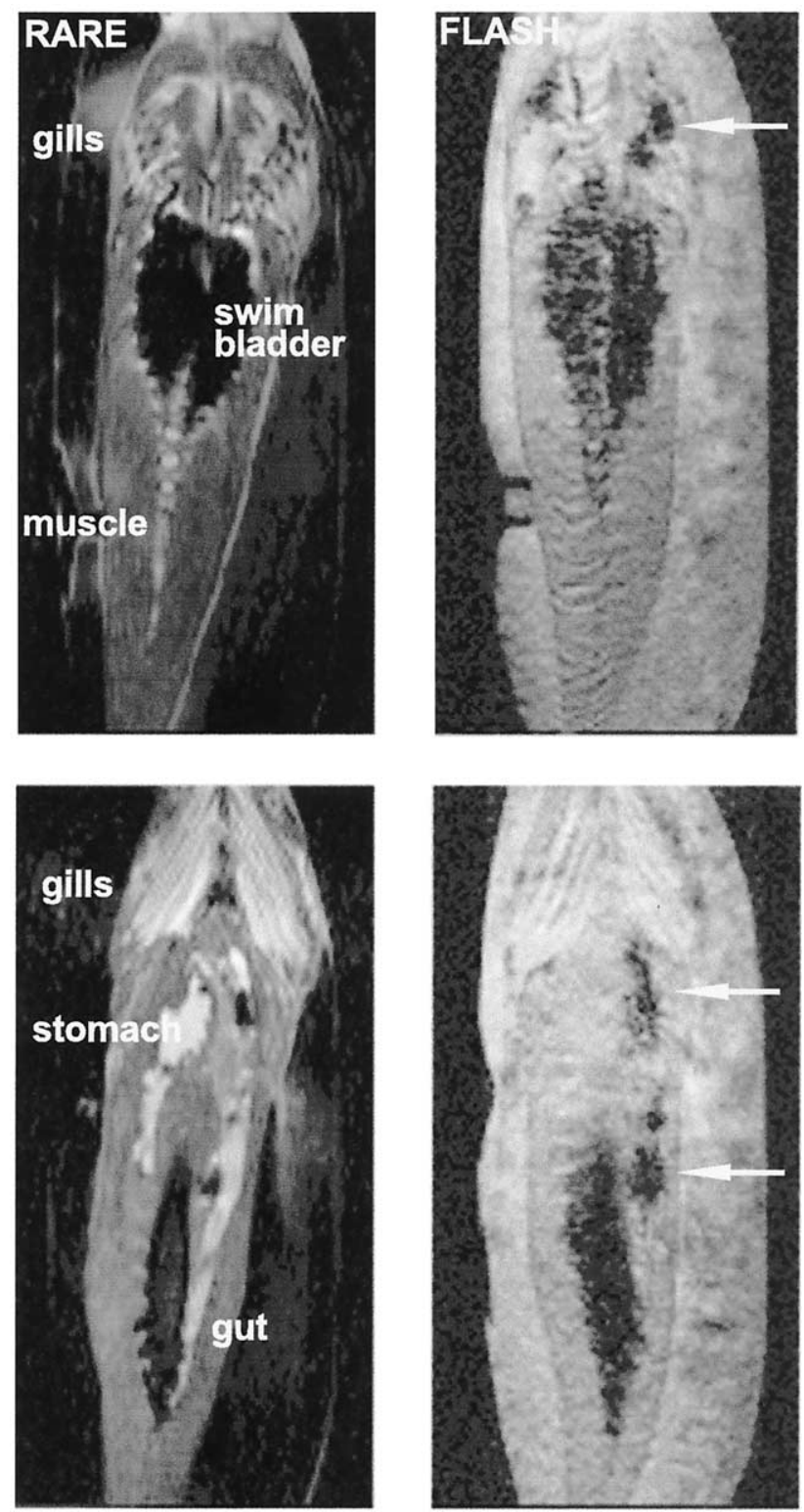

Fig. 1. Coronal MR imaging of cod Gadus morhua inside the animal chamber at a flow speed of $1.2 \mathrm{l} / \mathrm{min}$ obtained with a spin echo sequence (RARE) in comparison to a gradient echo sequence (FLASH). The gradient echo images showed strong movement artefacts throughout the whole images arising from water flow, whereas no residual water signal could be detected with the spin echo method. Consequently, no movement artefacts occurred during image acquisition resulting in a higher $\mathrm{S} / \mathrm{N}$ ratio from the fish. Note the good anatomical resolution of different organs inside the fish in contrast to the gradient echo image. Furthermore, susceptibility artefacts appeared in the gradient echo image (see arrows) (for imaging parameters see text).

its position and no movement artefacts could be observed during data acquisition (Fig. 2). The position of the animal in the chamber is typical for these sessile benthic animals and can frequently be observed in the aquarium system.

Owing to the long-term maintenance of this posture it was possible even to perform localized ${ }^{1} \mathrm{H}-\mathrm{NMR}$ spectroscopy. Fig. 3 presents a coronal anatomical image through
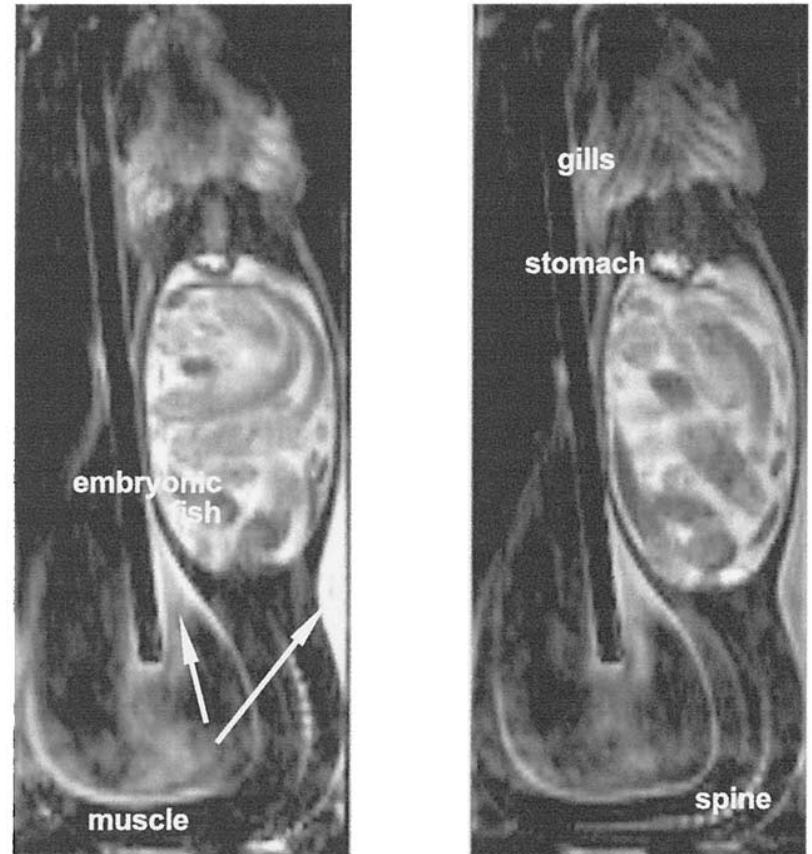

Fig. 2. In vivo MR images from a pregnant female of the viviparous eelpout Zoarces viviparus. Most obviously the uterus with a couple of embryonic fish is visible inside the fish, whereas the empty stomach resumed only a very small body part. Other tissues are identified as: muscle tissue, gills and spine. (Images obtained with RARE, parameters: matrix: $256 \times 192$, FOV $=12 \mathrm{~cm}^{2}, 4$ slices with a slice thickness of $1 \mathrm{~mm}$, TR $=2000 \mathrm{~ms}$, $\mathrm{TE}=10 \mathrm{~ms}$, a rare factor of 8 and a scan time of $1.5 \mathrm{~min}$ ). The arrows indicate regions of reduced water flow.

the uterus of the eelpout indicating two voxel positions for localized spectroscopy. One voxel was chosen in the uterus lumen of the animal, a second voxel in an embryonic fish. The spectra obtained from these voxels are presented in Fig. 3. The spectrum obtained from the amniotic fluid (B) showed almost no signal from metabolites except for the water signal, which resulted from imperfect water suppression. This indicated sufficient localisation. On the other hand, different signals could be detected in the spectrum obtained from embryonic fish (A): ATP, glycine (Gly), creatine/phosphocreatine $(\mathrm{Cr})$ and large signals from lipids (Lip). In combination with the long-term stability of the set-up these methods offer the opportunity to study noninvasively adult and embryonic animals in vivo (see also Fig. 5). The example clearly shows the applicability of different sophisticated MR methods in highly conductive media to investigate sessile animals without the need of anaesthesia or fixation.

An example for the application of dynamic MR imaging in marine fish is presented in Fig. 4. Flow weighted MR imaging was used to study the effect of temperature on circulatory performance of Baltic Sea eelpout. Flow weighted MR can be used for angiography and to observe blood flow changes in bigger vessels even in marine animals [3,5]. Fig. 4 shows a transversal flow weighted MR image through an eelpout at different temperatures (position of the slice indicated in the 


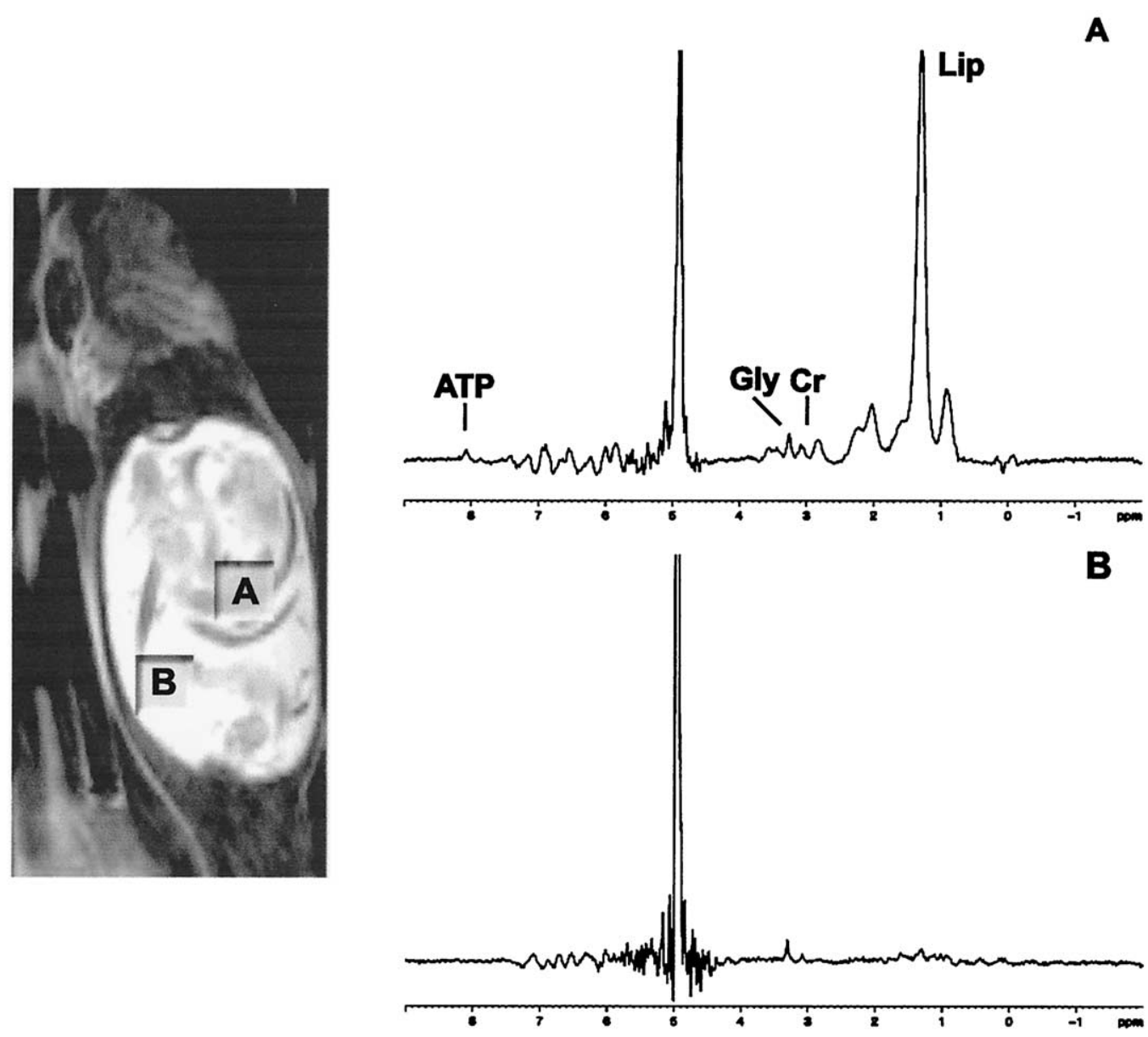

Fig. 3. In vivo localized ${ }^{1} \mathrm{H}-\mathrm{NMR}$ spectra collected in the same animal as in Fig. 2. Voxel locations are shown in the MR image on the left, fish embryo (A) and the lumen of the uterus (B). Signals identified in the embryo spectrum include ATP, glycine (Gly) and creatine (Cr) as well as large signals from lipids. Almost no signal could be detected in voxel B, indicating sufficient localisation. (for parameters see text).

anatomical coronal view). The bright spots arose from blood flowing through dorsal aorta and vein of the animal. Smaller vessels of the liver could also be observed. An increase in signal intensity indicating a rise in blood flow could be observed in the MR images at $18^{\circ} \mathrm{C}$ (see arrows). After 19 hours at a temperature of $22^{\circ} \mathrm{C}$ signal intensity in the blood vessels decreased. This indicates that animals have surpassed the socalled critical temperature, where main organ functions like circulatory flow started to collapse owing to temperature induced oxygen deficiency (see [11] for review). No artefacts from water flow or other susceptibility distortions could be observed in the images, due to the vertical direction of the phase gradients in relation to the direction of water flow. Fig. 5 presents consecutive localized ${ }^{1} \mathrm{H}-\mathrm{NMR}$ spectra obtained from a voxel located in the liver of the animal. Water suppression and signal line width were sufficient to resolve for typical metabolites like glycine (Gly), choline (Cho), creatine/phosphocreatine $(\mathrm{Cr})$. At higher temperatures $\left(\mathrm{T}=22^{\circ} \mathrm{C}\right)$ a signal increased at $1.3 \mathrm{ppm}$ indicating anaerobic metabolism by the production of lactate (Lac), concomitant with the reduction in blood flow, especially through the liver vessels of the animal (Fig. 4).

\subsection{Pelagic fish}

MR imaging techniques with standard sequences like RARE and FLASH could not be applied to unrestrained pelagic fish without movement artefacts, except in Fig. 1. In this case the fish was somewhat bigger than the animal chamber and its tail was slightly bent. The animal stopped swimming and it was possible to carry out MR imaging experiments without artefacts resulting from spontaneous movements. Nevertheless, since this does not reflect normal behaviour of a pelagic fish, further methodological work is required for the use with unrestrained pelagic animals. While imaging and localized spectroscopy experiments could not be carried out with pelagic swimming fish, in vivo ${ }^{31} \mathrm{P}-\mathrm{NMR}$ spectroscopy was unaffected by spontaneous movements of the animal. In Fig. 6 a stack plot of in vivo 

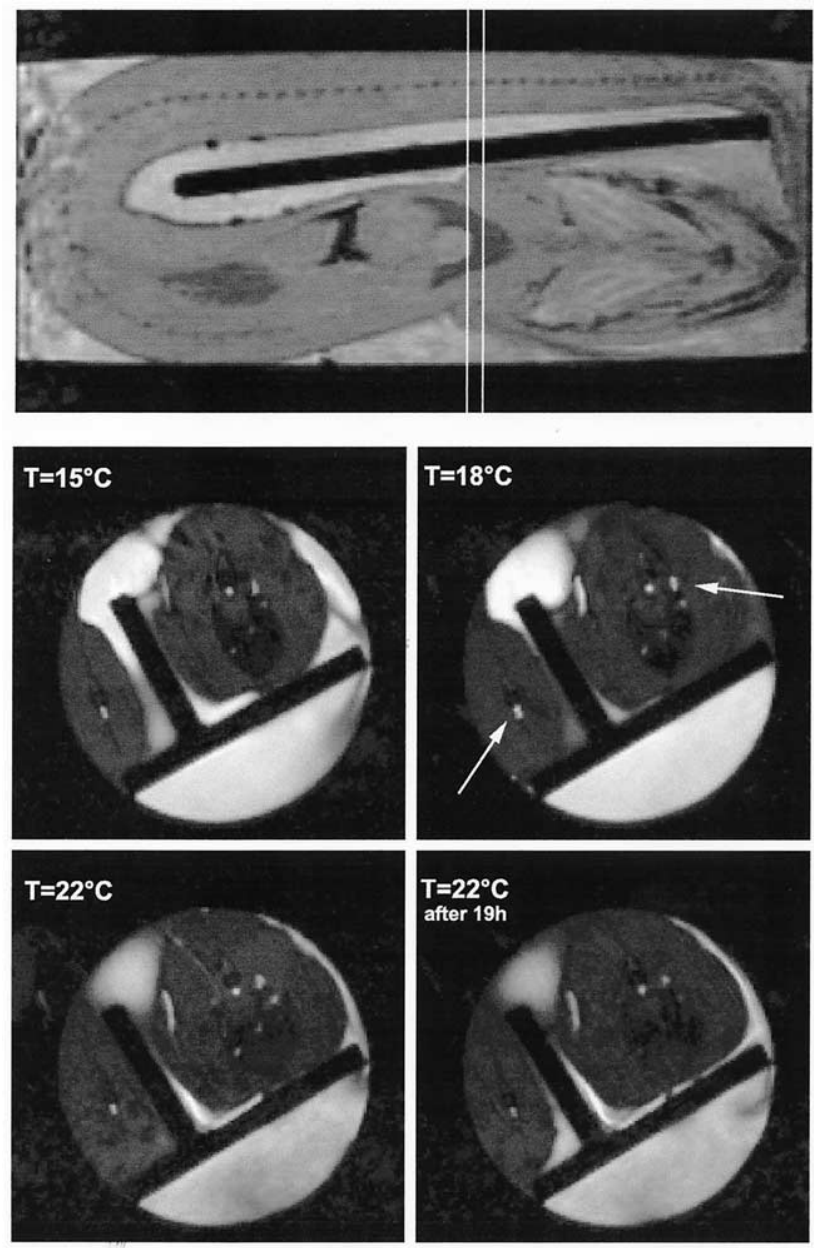

Fig. 4. Transversal flow compensated gradient echo images through the mid section of a Baltic Sea eelpout at different temperatures (slice position and thickness indicated in the coronal view of the animal). Blood flow through different vessels (Aorta dorsalis, Vena cava posterior located in the centre of the fish and three vessels through the liver) could be clearly visualized as bright spots inside the animal. After a slight increase in signal intensity at $18^{\circ} \mathrm{C}$ (see arrows), signal intensity in the vessels decreased after $19 \mathrm{~h}$ at $\mathrm{T}=22^{\circ} \mathrm{C}$, reflecting a reduction in blood flow, especially in the liver vessels where blood flow almost collapsed (black area) (for imaging parameters see 2. NMR methods).

${ }^{31} \mathrm{P}-\mathrm{NMR}$ spectra is presented which was collected in cod during and after a hypoxic challenge. Spectra were collected consecutively, each being accumulated over $5 \mathrm{~min}$. After recording control spectra (control, Fig. 6), hypoxia was induced by stopping water flow through the animal chamber (hypo). An increase in inorganic phosphate $(\mathrm{Pi})$ and a decrease in phosphocreatine ( $\mathrm{PCr})$, as well as a shift of the inorganic phosphate signal, reflecting intracellular acidification, could be detected in the subsequent spectra, indicating the onset of hypoxia for the fish inside the animal chamber. After a decrease in the $\beta$-ATP signal had been observed, indicating severe depletion of energy stores, flow rate of about $1 \mathrm{l} / \mathrm{min}$ was resumed. Directly after the onset of water flow energy metabolism started to recover, evidenced by a decrease in inorganic phosphate and an increase in phosphocreatine levels. After $15 \mathrm{~min}$ the animal was fully recovered indicated by the reestablish-
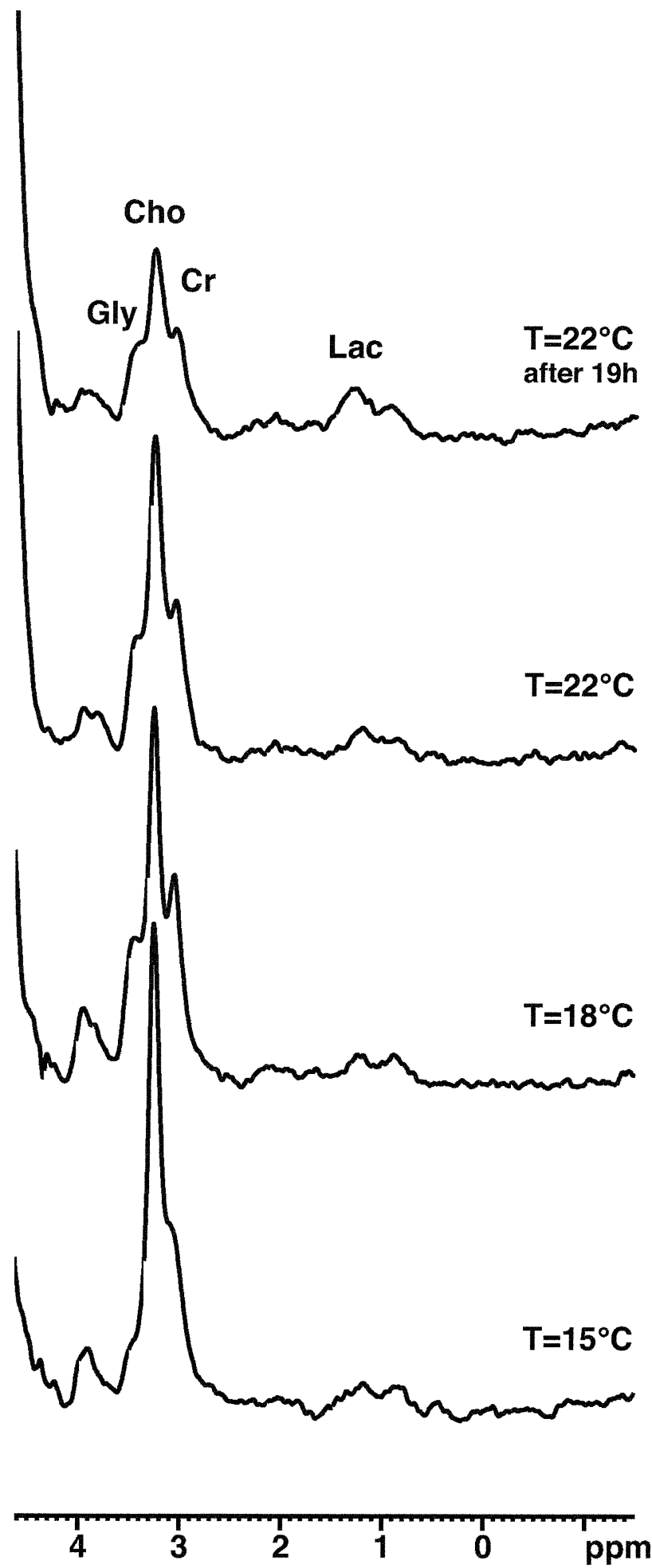

Fig. 5. In vivo localized ${ }^{1} \mathrm{H}-\mathrm{NMR}$ spectra from the liver of the same animal. Typical metabolites like glycine (Gly), choline (Cho), creatine/phosphocreatine $(\mathrm{Cr})$ could be detected. At higher temperatures $\left(\mathrm{T}=22^{\circ} \mathrm{C}\right)$ the lactate signal (Lac) at $1.3 \mathrm{ppm}$ increased indicating the onset of anaerobic metabolism, in accordance with the reduction in blood flow seen in Fig. 4.

ment of control spectra. This example shows that even in pelagic fish changes in metabolism could be monitored with in vivo NMR spectroscopy on a satisfactory time scale. 


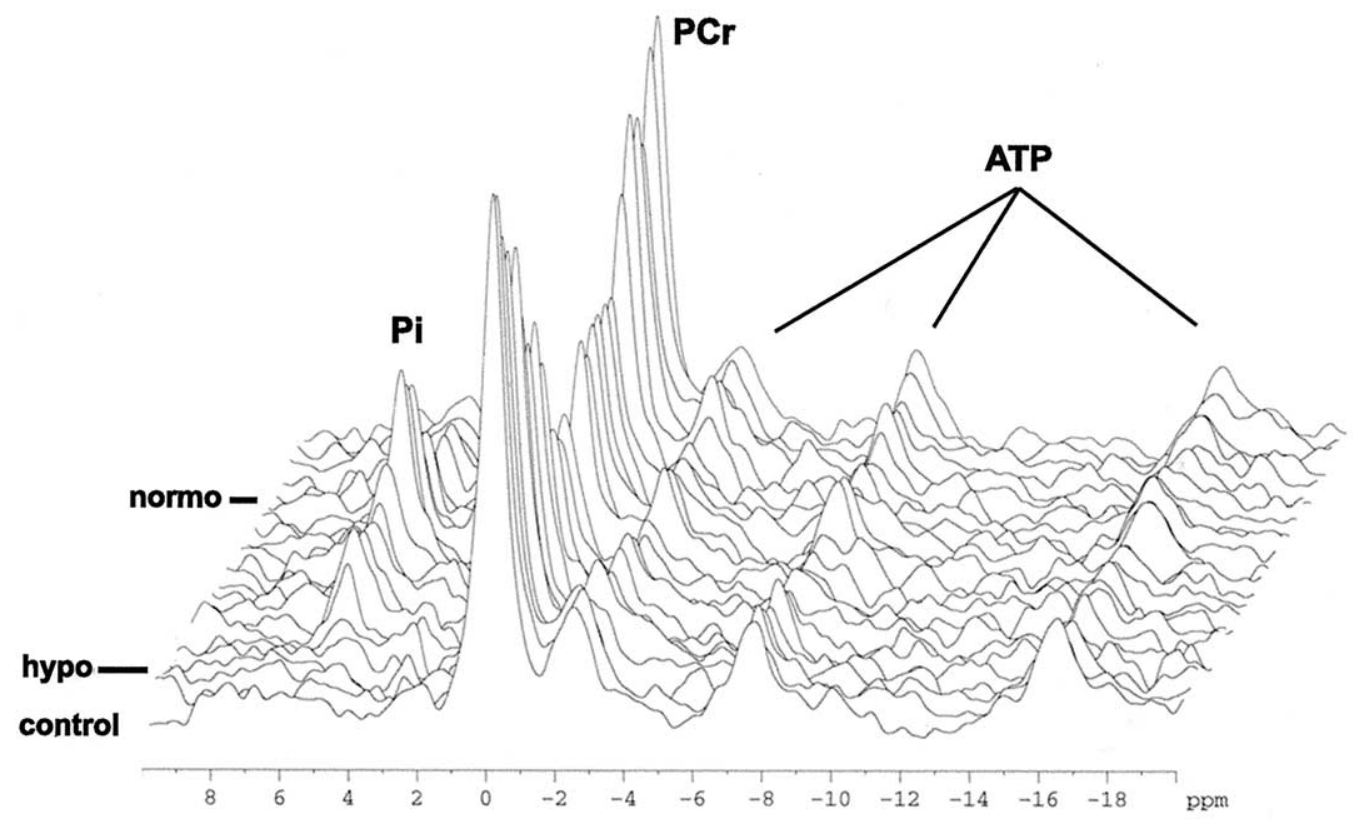

Fig. 6. Stack plot of in vivo ${ }^{31} \mathrm{P}-\mathrm{NMR}$ spectra from the muscle of cod G. morhua. Each spectrum was acquired over 5 min. Hypoxia (hypo) induced an increase in inorganic phosphate levels (Pi) accompanied by a depletion of phosphocreatine (PCr). After returning to control conditions the PCr signal increased and the Pi signal vanished indicating energetic recovery of the animal.

In conclusion, the development of faster and more stable MR methods with enhanced resolution for contrast information based on the development of improved hardware, allows the application of dynamic NMR imaging and spectroscopy methods to marine fish without the need of anaesthesia. Metabolic and functional aspects can be studied in these marine animals in addition to those described in Blackband and Stoskov [4]. MR spectroscopy and imaging may have a wide range of applications in the fields of comparative physiology, ecophysiology, natural products research etc. and will be a helpful tool for a better understanding of marine life and the scope of physiological adaptations to environmental factors and their fluctuations. This has become important especially with respect to recent investigations of the physiological basis of temperature dependent biogeography and a cause and effect understanding of the influence of climate change on the well-being and survival of fish and invertebrate species (e.g. [13]).

\section{Acknowledgments}

We like to thank Dipl. Ing. R.-M. Wittig for excellent technical assistance. Support by a BMBF grant (project 03PL02A, NMR laboratory) is gratefully acknowledged. A contribution to the ELOISE project: Effects of climateinduced change on marine coastal fishes (CLICOFI, ENV4CT97-0596) funded by the European Union. ELOISE publication No.

\section{References}

[1] Van den Thillart G, van Waarde A. Nuclear magnetic resonance spectroscopy of living systems: applications in comparative physiology. Physiol Rev 1996;76:799-837.

[2] Van der Linden A, Verhoye M, Nilsson GE. Does anoxia induce cell swelling in carp brains? In vivo MRI measurements in crucian carp and common carp. J Neurophysiol 2001;85:125-33.

[3] Fernández M, Bock C, Pörtner HO. The cost of being a caring mother: the ignored factor in the reproduction of marine invertebrates. Ecol Letters 2000;3:487-94.

[4] Blackband SJ, Stoskopf MK. In vivo nuclear magnetic resonance imaging and spectroscopy of aquatic organisms. Magn Reson Imaging 1990;8:191-8.

[5] Bock C, Frederich M, Wittig RM, Pörtner HO. Simultaneous observation of haemolymph flow and ventilation in marine spider crabs at different temperatures: a flow weighted MRI study. Magn Reson Imaging 2001;19:1113-24.

[6] Bock C, Sartoris FJ, Wittig RM, Pörtner HO. Temperature dependent $\mathrm{pH}$ regulation in stenothermal Antarctic and eurythermal temperate eelpout (Zoarcidae): an in vivo NMR study. Polar Biol 2001;24:86974.

[7] Sokolova IM, Bock C, Pörtner HO. Resistance to freshwater exposure in White Sea Littorina spp. II: acid base regulation. J Comp Physiol B 2000;170(2):105-15.

[8] Pörtner HO, Peck L, Zielinski S, Conway LZ. Intracellular pH and energy metabolism in the highly stenothermal Antarctic bivalve $\mathrm{Li}$ mopsis marionensis as a function of ambient temperature. Polar Biol 1999;22:17-30.

[9] Kugel H. Improving the signal-to-noise ratio of NMR signals by reduction of inductive losses. J Magn Reson 1988;91:179-85.

[10] Hennig J, Nauerth A, Friedburg H. RARE imaging: a fast imaging method for clinical MR. Magn Reson Med 1986;3:823-33. 
[11] Haase A, Frahm J, Matthaei D, Hänicke W, Merboldt K-D. FLASH imaging. Rapid NMR imaging using low flip-angle pulses. J Magn Reson 1986;67:258-66.

[12] Pörtner HO. Climate change and temperature-dependent biogeography: oxygen limitation of thermal tolerance in animals. Naturwissenschaften 2001;88:137-46.

[13] Pörtner HO, Berdal B, Blust R, Brix O, Colosimo A, De Wachter
B, Guiliani A, Johansen T, Fischer T, Knust R, Lannig G, Naevdahl G, Nedenes A, Nyhammer G, Sartoris FJ, Serendero I, Sirabella $\mathrm{P}$, Thorkildsen S, Zakhartsev M. Climate induced temperature effects on growth performance, fecundity and recruitment in marine fish: developing a hypothesis for cause and effect relationships in Atlantic cod (Gadus morhua) and common eelpout (Zoarces viviparus) Continental Shelf Res 2001;21:1975-97. 